\title{
Guest editorial: special section on artificial intelligence for requirements engineering
}

\author{
Eduard C. Groen ${ }^{1} \cdot$ Rachel Harrison ${ }^{2} \cdot$ Pradeep K. Murukannaiah $^{3}$. \\ Andreas Vogelsang ${ }^{4}$
}

Published online: 31 July 2019

○ Springer Science+Business Media, LLC, part of Springer Nature 2019

This section of the Automated Software Engineering journal is devoted to the theme of Artificial Intelligence for Requirements Engineering. The synergies between these two domains bear the potential to improve requirements quality, among other things through automated models, natural language processing, machine learning, reasoning about uncertainties and ambiguities, knowledge acquisition and representation, agent based solutions, problem solving and decision making support mechanisms, optimization techniques and automated approaches for prioritization.

Eight papers were received; five following an open call for papers, and three in response to targeted invitations to authors to submit an extended version of their accepted papers to the fifth Artificial Intelligence for Requirements Engineering (AIRE'18) workshop. The articles have undergone rigorous peer-review according to the journal's high standards, following which two papers were accepted.

The two articles that comprise this special issue contain a common thread of employing techniques from Artificial Intelligence, such as algorithms or natural language processing, to automate the identification of aspects relevant to a requirements engineer. They are briefly discussed as follows:

\footnotetext{
$凶 \quad$ Rachel Harrison

rachel.harrison@brookes.ac.uk

Eduard C. Groen

eduard.groen@iese.fraunhofer.de

Pradeep K. Murukannaiah

pkmvse@rit.edu

Andreas Vogelsang

andreas.vogelsang@tu-berlin.de

1 Fraunhofer IESE, Fraunhofer-Platz 1, 67663 Kaiserslautern, Germany

2 Oxford Brookes University, Wheatley Campus, Oxford OX33 1HX, UK

3 Software Engineering, GCCIS, Rochester Institute of Technology, 134 Lomb Memorial Dr., Room 70-1521, Rochester, NY 14623, USA

4 TU Berlin, Ernst-Reuter-Platz 7, 10587 Berlin, Germany
} 
The first article, by Davide Dell'Anna, Fabiano Dalpiaz, and Mehdi Dastani on "Requirements-driven Evolution of Sociotechnical Systems via Probabilistic Reasoning and Hill Climbing" proposes a framework to support the runtime validation of requirements models and to guide the evolution of a sociotechnical system. The manual evolution is analyst-driven, while the automated evolution revises requirements by a hill climbing algorithm. The approach is an effective way of adjusting a requirements model.

In the second article, "An NLP Approach for Cross-domain Ambiguity Detection in Requirements Engineering" by Alessio Ferrari and Andrea Esuli, the authors present a natural language processing approach that compares word embeddings from multiple domain-specific language models. By measuring differences in ways a term is used, ambiguous terms are identified that may not have the same meaning as in another domain. Addressing these ambiguities helps improve the shared understanding of stakeholders.

The special section was preceded by the AIRE'18 workshop, held on August 21st, 2018 at the 26th IEEE International Requirements Engineering Conference (RE'18) in Banff, Canada.

Together, these two papers illustrate the potential Artificial Intelligence has in helping to solve problems encountered in the field of Requirements Engineering. The quality of the research presented here suggests bright perspectives for the future of this intersection of domains.

The guest editors would like to thank the authors for their contributions, the reviewers for their constructive criticism and detailed comments, and the Editor-in-Chief as well as the editorial staff at Springer for their assistance during the review process.

Enjoy reading,

Eduard C. Groen, Rachel Harrison, Pradeep K. Murukannaiah, and Andreas Vogelsang July 5th, 2019

Publisher's Note Springer Nature remains neutral with regard to jurisdictional claims in published maps and institutional affiliations. 